\title{
Low frequency observations of the radio nebula produced by the giant flare from SGR 1806-20
}

\section{Polarimetry and total intensity measurements}

\author{
H. Spreeuw, B. Scheers and R. A. M. J. Wijers \\ Astronomical Institute "Anton Pannekoek", University of Amsterdam, Kruislaan 403, 1098 SJ Amsterdam, The Netherlands \\ e-mail: [j.n.spreeuw; l.h.a.scheers;r.a.m.j.wijers]@uva.nl
}

Received 30 September 2009 / Accepted 30 October 2009

ABSTRACT

\begin{abstract}
Context. The 2004 december 27 giant flare from SGR 1806-20 produced a radio nebula that was detectable for weeks. It was observed at a wide range of radio frequencies.

Aims. To investigate the polarized signal from the radio nebula at low frequencies and to perform precise total intensity measurements. Methods. We made a total of 19 WSRT observations. Most of these were performed quasi simultaneously at either two or three frequencies, starting 2005 january 4 and ending 2005 january 29. We reobserved the field in 2005 april/may, which facilitated an accurate subtraction of background sources.

Results. At $350 \mathrm{MHz}$, we find that the total intensity of the source is lower than expected from the GMRT $240 \mathrm{MHz}$ and $610 \mathrm{MHz}$ measurements and inconsistent with spectral indices published previously. Our $850 \mathrm{MHz}$ flux densities, however, are consistent with earlier results. There is no compelling evidence for significant depolarization at any frequency. We do, however, find that polarization angles differ substantially from those at higher frequencies.

Conclusions. Low frequency polarimetry and total intensity measurements provide a number of clues with regard to substructure in the radio nebula associated with SGR 1806-20. In general, for a more complete understanding of similar events, low frequency observations can provide new insights into the physics of the radio source.
\end{abstract}

Key words. stars: individual: SGR 1806-20 - stars: neutron - radio continuum: stars

\section{Introduction}

The 2004 december 27 flare from the Soft- $\gamma$-ray Repeater SGR 1806-20 was a major event in astronomy in a number of ways. First of all by the energy of the explosion: the brightest flash of radiation from beyond our solar system ever recorded. This is how it caught the attention of a larger audience. Secondly, because the flare provided new observational data about a known class of objects: magnetars, i.e., strongly magnetized neutron stars (see, e.g., Hurley et al. 2005). Also, it led to speculation about a possible link with $\gamma$-ray bursts (GRBs) (see, e.g., Tanvir et al. 2005). Theorists investigated the connection between the magnetic field and the explosion (see, e.g. Blandford 2005). Others focused on modeling the fireball and the afterglow (see, e.g., Nakar et al. 2005; Dai et al. 2005; Wang et al. 2005). Astronomers performed a number of follow-up observations at various wavelengths (Rea et al. 2005; Israel et al. 2005; Palmer et al. 2005; Schwartz et al. 2005; Fender et al. 2006). In particular, the flux from the radio nebula produced by the explosion (Gaensler et al. 2005a; Cameron et al. 2005; Taylor et al. 2005) was measured very frequently in 2005 january. These observations focused on total intensity measurements at various radio wavelengths and on polarimetry at $8.5 \mathrm{GHz}$. Some polarimetry was done at lower frequencies, but without the proper correction for the leakages (Gaensler et al. 2005b). We have performed accurate polarimetry at 350,850 and $1300 \mathrm{MHz}$. Also, we were able to measure the Stokes I flux from the radio nebula at 350 and $850 \mathrm{MHz}$ more precisely by observing the same field again in 2005 april/may. In this way, we could properly subtract the background sources from the $(u, v)$ data of the 2005 january observations. We compare our measurements with those at nearby frequencies.

\section{Observation and data reduction}

\subsection{General}

A total of 19 observations were performed in january, april and may of 2005. Four of these, on january 16, 20, 23 and 29 were alternating between 350 and $850 \mathrm{MHz}$. On january 7 and 10 scans at $1300 \mathrm{MHz}$ were also included. On january 4 we observed at 350, 650 and $1300 \mathrm{MHz}$, but the $650 \mathrm{MHz}$ data was not used. A summary is shown in Table 1.

We used AIPS (Greisen 2003) and ParselTongue (Kettenis et al. 2006) scripts for the reduction of all 19 datasets. The Westerbork Synthesis Radio Telescope (WSRT) was used for all observations. The WSRT is a linear array with 14 equatorially mounted 25-m dishes equipped with linear feeds. Its maximum baseline is $2.7 \mathrm{~km}$. All datasets recorded four polarization products with 8 IFs. 3C 286 was observed before the target and 3C 48 after. RFI was excised from the spectral line data using the AIPS task "SPFLG".

Calibration was done in four steps. First we determined the variation in system temperature as a function of time (and therefore also as a function of position on the sky), using the intermittent firing of a stable noise source. Next we performed 
Table 1. Summary of these 19 WSRT observations.

\begin{tabular}{cccc}
\hline \hline Epoch & $\begin{array}{c}\text { Days since } \\
\text { burst }\end{array}$ & $\begin{array}{c}\text { Time on } \\
\text { source }(\mathrm{min})\end{array}$ & $\begin{array}{c}\text { Frequency } \\
(\mathrm{MHz})\end{array}$ \\
\hline January 4 & 7.6 & 54 & 1300 \\
January 4 & 7.6 & 91 & 350 \\
January 5 & 8.6 & 462 & 850 \\
January 7 & 10.5 & 107 & 1300 \\
January 7 & 10.5 & 107 & 350 \\
January 7 & 10.5 & 107 & 850 \\
January 10 & 13.6 & 78 & 1300 \\
January 10 & 13.6 & 71 & 350 \\
January 10 & 13.6 & 71 & 850 \\
January 16 & 19.6 & 181 & 350 \\
January 16 & 19.6 & 181 & 850 \\
January 20 & 23.6 & 181 & 350 \\
January 20 & 23.6 & 165 & 850 \\
January 23 & 26.6 & 198 & 350 \\
January 23 & 26.6 & 198 & 850 \\
January 29 & 32.6 & 196 & 350 \\
January 29 & 32.6 & 196 & 850 \\
April 30/May 1 & 124.3 & 444 & 350 \\
May 2 & 125.2 & 464 & 850 \\
\hline
\end{tabular}

a bandpass calibration using the AIPS task "BPASS" using either 3C 48 or 3C 286 or both. We applied the bandpass solution using the AIPS task "SPLAT". After that, we performed an external absolute gain calibration using an assumed flux for 3C 48 by running the AIPS tasks "SETJY" and "CALIB". "SETJY" was set to use the absolute flux density calibration determined by Baars et al. (1977) and the latest (epoch 1999.2) polynomial coefficients for interpolating over frequency as determined at the VLA by NRAO staff. Finally, we self-calibrated the data for time variations in the relative complex gain phase and amplitude.

Polarization calibration was performed by running the AIPS task "LPCAL" on 3C 48 and "CLCOR" to correct for the instrumental XY phase offset. Generally, we followed the scheme for data reduction of WSRT data in AIPS as outlined by Robert Braun ${ }^{1}$, although we ran some AIPS tasks differently depending on frequency. Those differences mainly involved the details of polarization calibration. For instance, the leakage terms ("D terms") of the WSRT IFs are channel dependent, as pointed out by Brentjens (2008, paragraph 3.2). We took account of this, by first averaging groups of 5 channels through the AIPS task "SPLAT". Next, we ran "UVCOP" to make separate datasets from the averaged channels. After that, we ran "LPCAL" and "CLCOR" on each of these separately before applying the feed and XY instrumental phase offset corrections by again running "SPLAT".

Before imaging Stokes Q and Stokes U and before merging the datasets from 5 channel averaging back together through "DBCON", we applied a ParselTongue script for "derotation" to the residual data, i.e., the $(u, v)$ data where all sources except the target were removed, by running the AIPS task "UVSUB". The original Aips++ glish script was kindly given to us by G. Bernardi; we modified and translated it to a Python/ParselTongue script on a channel by channel basis. The "derotation" of the visibilities is absolutely necessary, since the rotation measure (RM) of SGR $1806-20$ is large, $272 \mathrm{rad} / \mathrm{m}^{2}$ (Gaensler et al. 2005a). This means that the polarized signal

\footnotetext{
1 See

http://www . astron.nl/radio-observatory/

astronomers/analysis-wsrt-data/

analysis-wsrt-dzb-data-classic-aips/analysis-wsrt-d
}

would vanish if all IFs were imaged simultaneously. For the $350 \mathrm{MHz}$ data, one really needs the derotation of the visibilities to be performed on a channel per channel basis, because the imaging of even one single IF would result in a severly corrupted measurement and underestimate of the fractional linear polarization. The uncertainty in this RM $\left(10 \mathrm{rad} / \mathrm{m}^{2}\right.$, see Gaensler et al. 2005a) is too large for accurate polarization angle measurements, especially at frequencies below $1 \mathrm{GHz}$. For this reason we determined the RM more accurately, by fitting the $\sin 2 \cdot \mathrm{RM} \cdot \lambda^{2}$ spectrum of either Stokes U or Stokes Q to its measured values at the 8 wavelengths $\lambda$ corresponding to the IFs near $350 \mathrm{MHz}$ and $850 \mathrm{MHz}$. The contribution to this RM from the ionosphere is naturally included in this fit, at least the part that did not vary during the observation run. We checked the output of the AIPS task "TECOR" for any significant variations in the ionospheric Faraday rotation during every observing run. The ionospheric Faraday rotation computed by "TECOR" is considered accurate since it does not use a model for the ionosphere but actual data from the CDDIS archive. We did not apply the ionospheric corrections from "TECOR" to our data because it implicitly assumes that one has recorded data from circular feeds.

It should be clear from Table 1 that the maximum observing time is $7.7 \mathrm{~h}$ due to the low declination of the source. Hence, the $(u, v)$ coverage is sparse for all observations, since linear arrays like the WSRT ideally have $12 \mathrm{~h}$ runs. The worst coverage was at three epochs when we alternated between three frequencies.

\subsection{Detailed desciption of the datasets}

\subsubsection{Observations at $350 \mathrm{MHz}$}

The $350 \mathrm{MHz}$ observations were performed on january 4, 7, 10, 16, 20, 23 and 29 and april 30/may 1 of 2005. The last observation was made to make an accurate subtraction of background sources possible. This mainly concerns the subtraction of the Luminous Blue Variable discussed in Supplementary Table 1 of Gaensler et al. (2005a). The time resolution of all observations, except the first and the last was $30 \mathrm{~s}$. On january 4 and april 30/may 1 the sampling times of the visibilities was $60 \mathrm{~s}$. The bandwidth per IF was $10 \mathrm{MHz}$, separated $8.75 \mathrm{MHz}$ from each other and centered on frequencies of $315.00,323.75,332.50$, $341.25,350.00,358.75,367.50$ and $376.25 \mathrm{MHz}$. The IFs were split into 64 channels, each $156.25 \mathrm{kHz}$ wide, except for the april 30/may 1 observation. For that observation, the IFs were split into 128 channels, each $78.125 \mathrm{kHz}$ wide. We used an automated flagger for the initial editing of our data: WSRT flagger ${ }^{2}$. 3C 286 was included in the external gain calibration, along with 3C 48. This was trivial, since 3C 286 is unpolarized at this frequency. The assumed fluxes for $3 \mathrm{C} 48$ and $3 \mathrm{C} 286$ in the lowest frequency IF were 43.889 and $26.106 \mathrm{Jy}$, respectively.

The april 30/may 1 observation has the best $(u, v)$ coverage. After performing 10 iterations of self calibration on this dataset the rms noise in the final image was $2.5 \mathrm{mJy} /$ beam. Its clean components were used to solve for the gain phases and amplitudes of the other datasets using a rather sophisticated scheme. First, a deconvolution of each of the 2005 january datasets was done in order to subtract the central region containing the radio nebula and the LBV, using the AIPS tasks "IMAGR", "CCEDT" and "UVSUB". The residual data were calibrated on the april 30/may 1 model which had the clean components from the central region removed. The gain phase and amplitude solutions were then copied and applied to the original

\footnotetext{
$\overline{2 \text { http://www.astron.nl } / \sim r e n t i n g / ~}$
} 
2005 january datasets. It this way we made sure that the Stokes I flux from SGR 1806-20 would not be reduced by calibrating on a model from an observation months after the flare. As explained in section 2.2.3, amplitude self calibration could also reduce the Stokes Q flux. However, due to the large RM of the source and because we use 45 of the available 64 channels, the Stokes Q flux almost completely vanishes in a single IF at $350 \mathrm{MHz}$. Thus this problem does not occur, at least not before "derotation".

PSR 1937+21 was observed in between SGR 1806-20 and 3C 48 for polarization calibration. This polarization calibration technique is decribed in detail by Brentjens (2008, paragraph 3.2). Since the RM of this pulsar is positive, Stokes Q should be $90^{\circ}$ ahead of Stokes $U$ with increasing $\lambda^{2}$, as noted by Brown \& Rudnick (2009, paragraph 2.3).

\subsubsection{Observations at $850 \mathrm{MHz}$ (“UHF high”)}

We observed SGR 1806-20 on january 5, 7, 10, 16, 20, 23, 29 and may 5 of 2005 . The last observation was performed to make an accurate subtraction of background sources possible. The time resolution of all observations, except for the first and the last, was $30 \mathrm{~s}$. The sampling time of the visibilities on january 5 and may 5 was $60 \mathrm{~s}$. The bandwidth of the eight IFs is $10 \mathrm{MHz}$, they were separated exactly $10 \mathrm{MHz}$ from each other and ranging from 805 to $875 \mathrm{MHz}$. Each IF was split in 64 channels with a width of $156.25 \mathrm{kHz}$, except for the may $1 / 2$ data that were split into 128 channels of $78.125 \mathrm{kHz}$. The external gain calibration was performed using an assumed flux for $3 \mathrm{C} 48$ of $24.240 \mathrm{Jy}$ for the lowest frequency IF. The $850 \mathrm{MHz}$ were reduced in almost the same way as the $1300 \mathrm{MHz}$ data. Only polarization calibration was performed slightly differently. Since the Stokes Q (and $\mathrm{U})$ of 3C 286 are not known for the "UHF high" frequencies, when the task "CALIB" was run on this calibrator, it was set to solve for gain phases only and not for gain amplitudes.

\subsubsection{Observations at $1300 \mathrm{MHz}$}

We observed SGR 1806-20 at $1300 \mathrm{MHz}$ on january 4,7 and 10 of 2005 . The total intensity measurements have already been published (see Gaensler et al. 2005a), so we focused on the polarized signal. However, we did check that our Stokes I fluxes agreed with those previously published.

On 2005 january 4 visibilities were recorded every $60 \mathrm{~s}$, on january 7 and 10 every $30 \mathrm{~s}$. The eight 20-MHz IFs were centered on frequencies of 1255, 1272, 1289, 1306, 1323, 1340 and $1357 \mathrm{MHz}$. Each IF was split in 64 channels with a width of $312.5 \mathrm{kHz}$. The external gain calibration was performed using an assumed flux for 3C 48 of $17.388 \mathrm{Jy}$ for the lowest frequency IF. 3C 286 was also included in the external gain amplitude and phase calibration using an assumed flux of 15.550 at $1255 \mathrm{MHz}$. 3C 286 is linearly polarized. We took account of this and of the usual "AIPS for linear feeds" projection $(\mathrm{R} \rightarrow \mathrm{X}, \mathrm{L} \rightarrow \mathrm{Y})$ by placing the assumed Stokes Q flux of 3C 286 (0.594 Jy at the lowest frequency IF) with a minus sign at the position of Stokes $\mathrm{V}$ in the AIPS SU table. For the other IFs we kept the same ratio between Stokes I and Stokes Q. In this way we could use 3C 286 not only for fixing the instrumental XY phase offset, but also for external gain calibration.

Self-calibration was run to solve for the gain phases only, since solving for the amplitudes could reduce the Stokes Q flux. The AIPS task "CALIB" cannot be set to run simultaneously on a Stokes I and Stokes Q model. Obviously, when "CALIB" is run on a Stokes I model, it implicitly assumes that $\mathrm{Q}=0$.
Table 2. Stokes I flux measurements at 350 and $850 \mathrm{MHz}$.

\begin{tabular}{|c|c|c|c|c|c|}
\hline \multirow[b]{2}{*}{$\begin{array}{l}\text { Epoch } \\
(2005 \\
\text { date })\end{array}$} & \multirow[b]{2}{*}{$\begin{array}{l}\text { Days } \\
\text { since } \\
\text { burst }\end{array}$} & \multicolumn{2}{|c|}{$350 \mathrm{MHz}$} & \multicolumn{2}{|c|}{$850 \mathrm{MHz}$} \\
\hline & & $\begin{array}{l}\text { Stokes I } \\
\text { flux dens. } \\
\text { mJy/beam }\end{array}$ & $\begin{array}{c}1 \sigma \\
\text { error } \\
\mathrm{mJy} / \text { beam }\end{array}$ & $\begin{array}{l}\text { Stokes I } \\
\text { flux dens. } \\
\text { mJy/beam }\end{array}$ & $\begin{array}{c}1 \sigma \\
\text { error } \\
\mathrm{mJy} / \text { beam }\end{array}$ \\
\hline Jan. 4 & 7.6 & 186 & 20 & & \\
\hline Jan. 5 & 8.6 & & & 157 & 10 \\
\hline Jan. 7 & 10.5 & 84 & 10 & 97 & 28 \\
\hline Jan. 10 & 13.6 & 78 & 10 & 50 & 22 \\
\hline Jan. 16 & 19.6 & 16 & 10 & 35 & 14 \\
\hline Jan. 20 & 23.6 & 7 & 10 & 21 & 8 \\
\hline Jan. 23 & 26.6 & 13 & 10 & 17 & 7 \\
\hline Jan. 29 & 32.6 & 1 & 10 & 22 & 9 \\
\hline
\end{tabular}

Clean components from the 2005 april 30/may 1 and may $1 / 2$ observations were subtracted.

Consequently, the same model is used to derive the $\mathrm{X}$ gains from the $\mathrm{XX}$ visibilities as the $\mathrm{Y}$ gains from the $\mathrm{YY}$ visibilities, while in fact $\mathrm{XX}=\mathrm{I}-\mathrm{Q}$ and $\mathrm{YY}=\mathrm{I}+\mathrm{Q}$, so different models should be used. When solving for gain phases only, the error made is generally considered acceptable.

\section{Results}

\subsection{Total intensity measurements}

The total intensity flux measurements at $350 \mathrm{MHz}$ were done by fitting a Gaussian of the same shape and size as the restoring beam to the (fixed) location of SGR 1806-20 in the Stokes I images. This was done by the AIPS task "IMFIT". We used the position from Gaensler et al. (2005a) ( $\left.\alpha=18 \mathrm{~h} 08 \mathrm{~m} 39.343 \mathrm{~s}, \delta=-20^{\circ} 24^{\prime} 39.8^{\prime \prime}\right)$ for the fits. The results are summarized in Table 2 . The error bars are conservative estimates from measurements of the residuals of bright sources in the field. The actual rms noise in these images is much lower, around $3 \mathrm{mJy} /$ beam, which is about the same as the error from "IMFIT".

\subsection{Polarimetry}

\subsubsection{General}

Polarimetry was performed on 2005 january 4, 5, 7 and 10 . Although all of our observations recorded full Stokes, we anticipated that it would not be possible to detect the polarized signal from SGR 1806-20 on later dates, since the total intensity drops rapidly. Also, we did not expect polarization fractions to exceed the values given by Taylor et al. (2005, Table 2).

\subsubsection{Determining the RM of SGR $1806-20$}

As noted before, the rotation measure (RM) as measured by (Gaensler et al. 2005a, $272 \pm 10 \mathrm{rad} / \mathrm{m}^{2}$ ) has a rather large error bar which translates into a polarization angle uncertainty at $1300 \mathrm{MHz}$ of $30.5^{\circ}$. At $850 \mathrm{MHz}$ this is even $71.4^{\circ}$. Naturally, the RM should be determined more accurately before polarization angles are to be measured.

This can be done by plotting Stokes U or Q fluxes of SGR 1806-20 as a function of frequency and fit for the RM. We are in the advantageous position that these WSRT observations were performed with eight IFs. Over a wide span of frequencies there are many turns of Stokes U (or Q) since its spectrum is sinusoidal as a function of $\lambda^{2}$. This effect is largest at low frequencies: at 
Table 3. RM measurements of SGR1806-20.

\begin{tabular}{ccccc}
\hline \hline $\begin{array}{c}\text { Epoch } \\
(2005 \\
\text { date })\end{array}$ & $\begin{array}{c}\text { Days } \\
\text { since } \\
\text { burst }\end{array}$ & $\begin{array}{c}\text { Frequency } \\
(\mathrm{MHz})\end{array}$ & $\begin{array}{c}\text { Measured } \\
\mathrm{RM} \\
\left(\mathrm{rad} / \mathrm{m}^{2}\right)\end{array}$ & $\begin{array}{c}1 \sigma \\
\text { error } \\
\left(\mathrm{rad} / \mathrm{m}^{2}\right)\end{array}$ \\
\hline Jan. 4 & 7.6 & 350 & 253.24 & 1.82 \\
Jan. 5 & 8.6 & 850 & 253.14 & 12.43 \\
Jan. 7 & 10.5 & 350 & 253.65 & 1.05 \\
Jan. 10 & 13.6 & 350 & 261.74 & 2.04 \\
\hline
\end{tabular}

$1300 \mathrm{MHz}$, there is less than one cycle of $A \cdot \sin \left(2 \cdot \mathrm{RM} \cdot \lambda^{2}+\phi\right)$, at $850 \mathrm{MHz}$ there are almost two cycles and at $350 \mathrm{MHz}$ there are 23 cycles. It is evident that the most accurate measurement can be made at the lowest frequency, if there is sufficient signal to noise. Fortunately, we could detect polarized signal at $350 \mathrm{MHz}$ from all three observations on 2005 january 4,7 and 10 after an initial "derotation" of our visibilities using the RM from Gaensler et al. (2005a, $272 \mathrm{rad} / \mathrm{m}^{2}$ ). This initial derotation prevents diminution of the polarized signal in a single IF. At $850 \mathrm{MHz}$ this initial derotation was not necessary. The noise levels at that frequency were such that detecting a polarized signal was only possible on 2005 january 5 and 7, but the latter observation yielded a very poor constraint on the RM, so we left it out. The $1300 \mathrm{MHz}$ data also gave very poor constraints on the RM, thus in determining the weighted mean RM we ignored those, too. For the other observations, we plotted Stokes U per IF and solved for the RM $(850 \mathrm{MHz})$ or the correction to the RM (350 MHz), as illustrated in Fig. 2. The results are shown in Table 3. It turned out that the noise levels in all of the Stokes Q maps were much higher than in the Stokes U maps, so we did not use them. In determining the weighted mean RM we also took into account the measurement by Gaensler et al. (2005a, $272 \pm 10 \mathrm{rad} / \mathrm{m}^{2}$ ) From this set of five measurements we derived an $\mathrm{RM}$ of $255.01 \pm 0.83 \mathrm{rad} / \mathrm{m}^{2}$. It should be clear that, with regard to the $350 \mathrm{MHz}$ RM measurements, the fits give the same reduced $\chi^{2}$ for both the positive and the negative correction to the initial "derotation". We removed those ambiguities by considering the Stokes U measurements near $850 \mathrm{MHz}$ data on 2005 january 5. The fit to that data gave an RM of $253.14 \pm 12.43 \mathrm{rad} / \mathrm{m}^{2}$ which made all of the positive RM solutions to the $350 \mathrm{MHz}$ data very unlikely $(\simeq 3.0 \sigma$ level for january 4 and 7$)$.

It is evident that the contribution of the ionosphere to the $\mathrm{RM}, \mathrm{RM}_{\mathrm{ion}}$, is included in all fits. For the 2005 january 4, 5, 7 and 10 observations, $\mathrm{RM}_{\mathrm{ion}}$ as reported by the AIPS task "TECOR", is the range $2.1 \pm 0.4 \mathrm{rad} / \mathrm{m}^{2}$. Consequently, the interstellar $\mathrm{RM}$ is given by $\mathrm{RM}_{\text {int }}=255.01-2.1=252.91 \pm$ $0.92 \mathrm{rad} / \mathrm{m}^{2}$.

\subsubsection{Polarization fractions and position angles}

We were able to measure the fractional linear polarization on all of the four epochs mentioned in paragraph 3.2.1. At $850 \mathrm{MHz}$, we were not able to measure polarization on 2005 january 10 . For the other occasions, the measured polarized fluxes, $P=$ $\sqrt{Q^{2}+U^{2}}$, fractions and their error bars are listed in Table 4. The latter two quantities are depicted in Fig. 3. The overall conclusion is that there is no compelling evidence for any significant depolarization at any frequency. Only the polarization fraction at $1300 \mathrm{MHz}$ on january 4 is low compared to the $8.4 \mathrm{GHz}$ measurements, but this fraction was determined from our worst fit, i.e., the fit with the highest reduced $\chi^{2}$.

The polarization angles and their uncertainties are also listed in Table 4. The observations at 850 and $1300 \mathrm{MHz}$ gave the

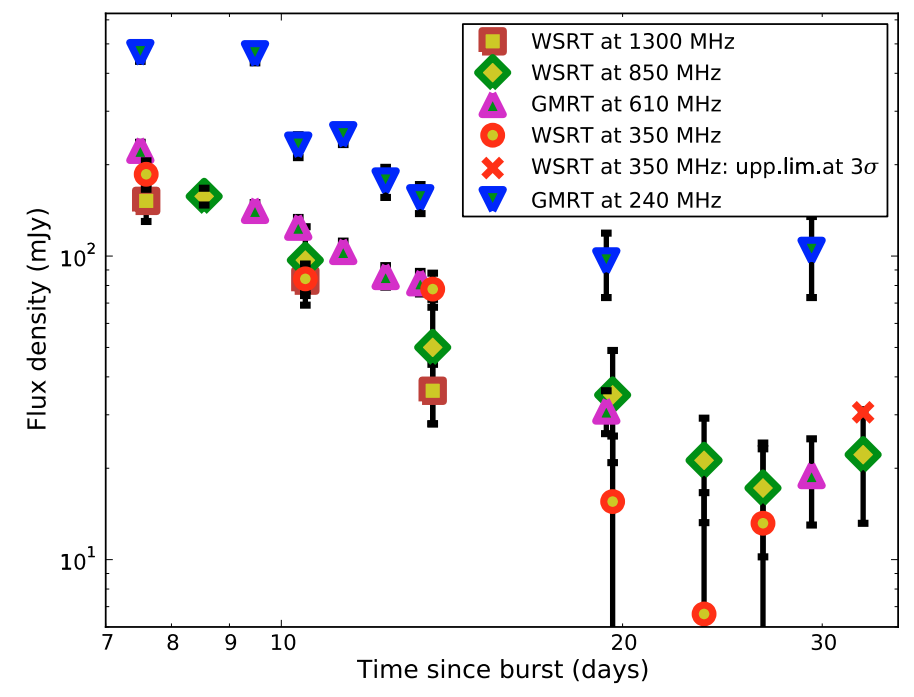

Fig. 1. Comparison between the 240,350, 610 and $1300 \mathrm{MHz}$ fluxes of the radio nebula associated with SGR 1806-20. The $1300 \mathrm{MHz}$ fluxes were published previously (Gaensler et al. 2005a). The fluxes at $350 \mathrm{MHz}$ and $1300 \mathrm{MHz}$ are approximately equal on 2005 january 7 , they coincide in this plot. For the $350 \mathrm{MHz}$ flux on 2005 january 29, instead of the flux, the $3 \sigma$ upper limit on the flux is depicted in order to enable a more appropriate vertical scale.

most accurate position angles, with typical uncertainties of order $10^{\circ}$. They are depicted in Fig. 4. Here, we see compelling evidence for significantly different polarization angles with respect to the $8.4 \mathrm{GHz}$ observations from Taylor et al. (2005), particularly on january 5 at $850 \mathrm{MHz}$ and on january 7 at both 850 and $1300 \mathrm{MHz}$.

\section{Discussion}

\subsection{Total intensity measurements}

It is clear from Fig. 1 that SGR1806-20 is much dimmer at $350 \mathrm{MHz}$ than what would be expected from the GMRT observations at 240 and $610 \mathrm{MHz}$ (Cameron et al. 2005). In principle the Luminous Blue Variable, 14" to the east of SGR 1806-20 (see the Supplementary Information to Gaensler et al. 2005a) should be easily distinguishable from the Soft Gamma Repeater in the GMRT images, even at $240 \mathrm{MHz}$. The FWHM beamsize reported at that frequency is $12^{\prime \prime} \times 18^{\prime \prime}$ (Chandra 2005b). This makes it hard to understand the discrepancy.

In principle the discrepancy cannot originate from the inclusion or exclusion of extended emission. The GMRT data were corrected for this (Chandra 2005a,b). We excluded short spacings $(<1 \mathrm{k} \lambda)$ from our $350 \mathrm{MHz}$ WSRT observations. This was actually a necessity since these were daytime observations and solar interference would otherwise compromise our calibration (see also Brentjens 2008, end of paragraph 3.2).

Also, it is possible that the LBV radio nebula is variable and that it was much brighter on 2005 april 30/may 1 than on some occasions in 2005 january. We ran the AIPS task "IMFIT" on the map from our 2005 april 30/may 1 observation and we found a peak flux density of $138 \pm 1 \mathrm{mJy} /$ beam and an integrated flux of $189 \pm 2 \mathrm{mJy} /$ beam at the location of the LBV. The NVSS (Condon et al. 1998) image of this field shows this source at the $15 \mathrm{mJy}$ level. This would indicate that the LBV has a spectral index of about -1.8 , which is almost the index for thermal radio radiation. It should be noted that, at the times of the latest observations in january 2005 , when the radio nebula was relatively 
Table 4. Polarimetric measurements of SGR 1806-20.

\begin{tabular}{cccccccccc}
\hline \hline $\begin{array}{c}\text { Epoch } \\
(2005)\end{array}$ & $\begin{array}{c}\text { Days since } \\
\text { burst }\end{array}$ & $\begin{array}{c}\text { Frequency } \\
(\mathrm{MHz})\end{array}$ & $\begin{array}{c}\sqrt{Q^{2}+U^{2}} \\
(\mathrm{mJy} / \text { beam })\end{array}$ & $\begin{array}{c}1 \sigma \\
\text { error }(\mathrm{mJy} / \text { beam })\end{array}$ & $\begin{array}{c}\text { Polarization } \\
\text { fraction }(\%)\end{array}$ & $\begin{array}{c}1 \sigma \\
\text { error }(\%)\end{array}$ & $\begin{array}{c}\text { Polarization } \\
\text { angle }\left({ }^{\circ}\right)\end{array}$ & $\begin{array}{c}1 \sigma \\
\text { error }\left({ }^{\circ}\right)\end{array}$ & $\begin{array}{c}\text { Reduced } \\
\chi^{2}\end{array}$ \\
\hline Jan. 4 & 7.6 & 350 & 2.68 & 0.81 & 1.44 & 0.46 & 103 & 39 & 0.70 \\
Jan. 4 & 7.6 & 1300 & 0.71 & 0.66 & 0.47 & 0.44 & 31 & 26 & 1.571 \\
Jan. 5 & 8.6 & 850 & 2.22 & 0.28 & 1.41 & 0.20 & 96 & 7 & 0.85 \\
Jan. 7 & 10.5 & 350 & 2.30 & 0.64 & 2.73 & 0.82 & 69 & 38 & 0.33 \\
Jan. 7 & 10.5 & 850 & 1.71 & 0.52 & 1.76 & 0.74 & 44 & 9 & 0.65 \\
Jan. 7 & 10.5 & 1300 & 1.90 & 0.37 & 2.29 & 0.48 & 50 & 7 \\
Jan. 10 & 13.6 & 350 & 1.14 & 0.87 & 1.46 & 1.13 & 36 & 47 \\
Jan. 10 & 13.6 & 1300 & 1.31 & 0.40 & 3.65 & 1.38 & 69 & 1.06 \\
\hline
\end{tabular}

Notes. ${ }^{(1)}$ Poor fit.

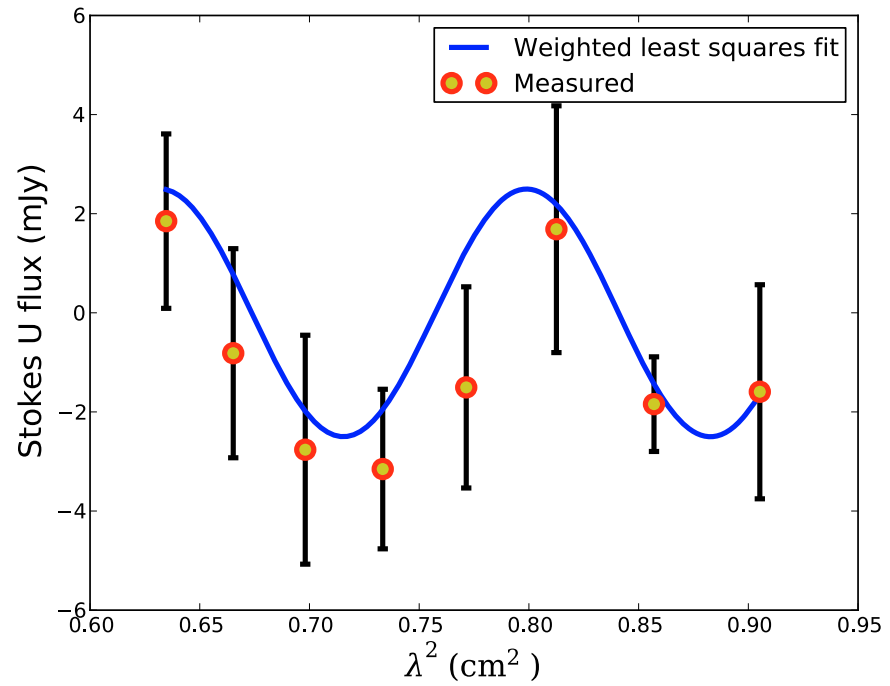

Fig. 2. Determining the rotation measure of SGR $1806-20$ by fitting the sinusoidal Stokes U spectrum. Here, the fit was made to the values of Stokes U on 2005 january 04 at the wavelengths corresponding to the $8 \mathrm{IFs}$ near $350 \mathrm{MHz}$ after the visibilities were "derotated" by an angle corresponding to an $\mathrm{RM}$ of $-272 \pm 10 \mathrm{rad} / \mathrm{m}^{2}$. We used gnuplot to fit the function $A \cdot \sin \left(2 \cdot \mathrm{RM} \cdot \lambda^{2}+\theta\right)$ for three free parameters $A, \mathrm{RM}$ and $\theta$. The correction to the RM from this fit is $-18.76 \pm 1.82 \mathrm{rad} / \mathrm{m}^{2}$. The reported reduced $\chi^{2}$ is 0.69 .

dim, there is no evidence for negative residuals in our maps that could be caused by the subtraction of the LBV. This indicates that, most likely, the LBV had the same brightness at the times of at least some of the 2005 january measurements as on 2005 april 30/may 1.

Variability at radio wavelengths of the radio nebulas from LBVs has been known for quite some time (see, e.g., Abbott et al. 1981). For the P Cygni nebula variability at timescales of days was established at cm wavelengths (Skinner et al. 1996). These authors report a 50\% increase in flux in less than two days on one occasion during three months of observations on every other day. It is unknown how these variations translate to lower frequencies. We therefore cannot completely exclude that the LBV was brighter at the time of the 2005 april 30/may 1 observation than on some occasions in january 2005. Also, the spectral index derived above does not agree with any of the spectral indices of the four LBVs observed by Duncan \& White (2002) at 3 and $6 \mathrm{~cm}$. Two of those spectral indices are close to that of a spherically symmetric radially expanding stellar wind $(+0.6$, see Panagia \& Felli 1975; Wright \& Barlow 1975). However, at these wavelengths, those systems may well be described as

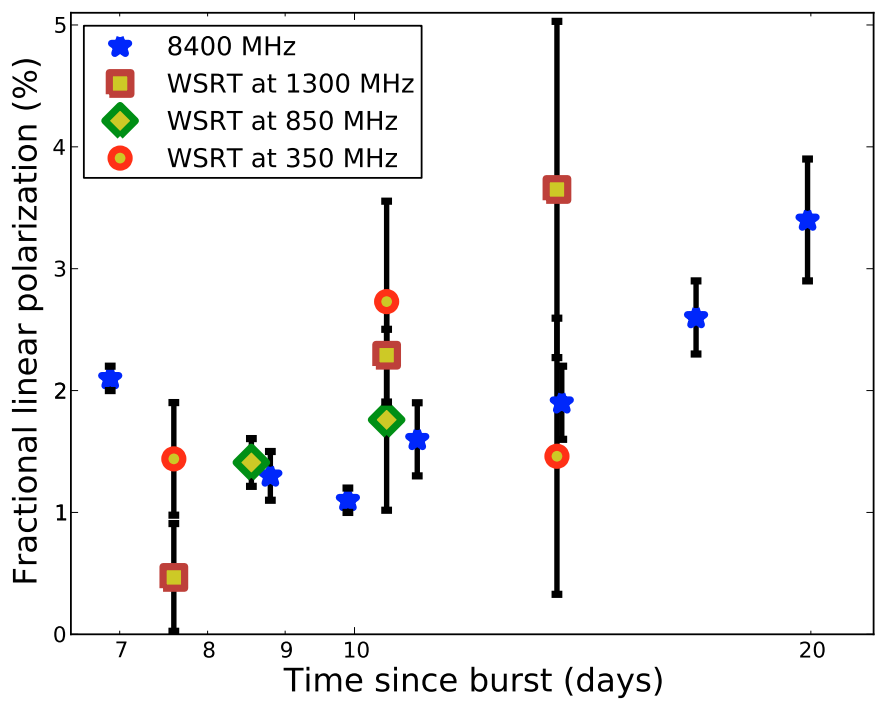

Fig. 3. Comparison between linear polarization fractions at 350, 850, 1300 and $8400 \mathrm{MHz}$

optically thin, which may not be the case at the frequencies we are considering.

The WSRT $850 \mathrm{MHz}$ Stokes I measurements are not inconsistent with the $840 \mathrm{MHz}$ MOST data published earlier (Gaensler et al. 2005a), given the rather large noise levels in the data from both telescopes. The last MOST observation was taken 15 days after the Giant Flare (GF). Consequently, the $850 \mathrm{MHz}$ WSRT observations after 2005 january 10 cannot be compared with other observations in this band. The last three of the january 2005 observations at $850 \mathrm{MHz}$ were less contaminated by RFI than the first four, which resulted in smaller error bars on the fluxes. There is evidence ( $>2 \sigma$ level) for a deviation from a power-law decay from about 15 days after the GF, analogous to the $4.8 \mathrm{GHz}$ observations by Gelfand et al. (2005, paragraph 2). These authors also mention a gradual rebrightening from about 25 days after the GF, as a result of swept up ambient material. We can also see that in the WSRT $850 \mathrm{MHz}$ data, but the evidence for this is less compelling, since the sampling of these observations is sparse in time. Consequently, it is shown only in one of our observations, on 2005 january 29, 32.6 days after the GF.

\subsection{Polarimetric measurements}

In Fig. 3 we compare the polarization fractions as listed in Table 4 with the measurements at $8.4 \mathrm{GHz}$ by Taylor et al. (2005, 


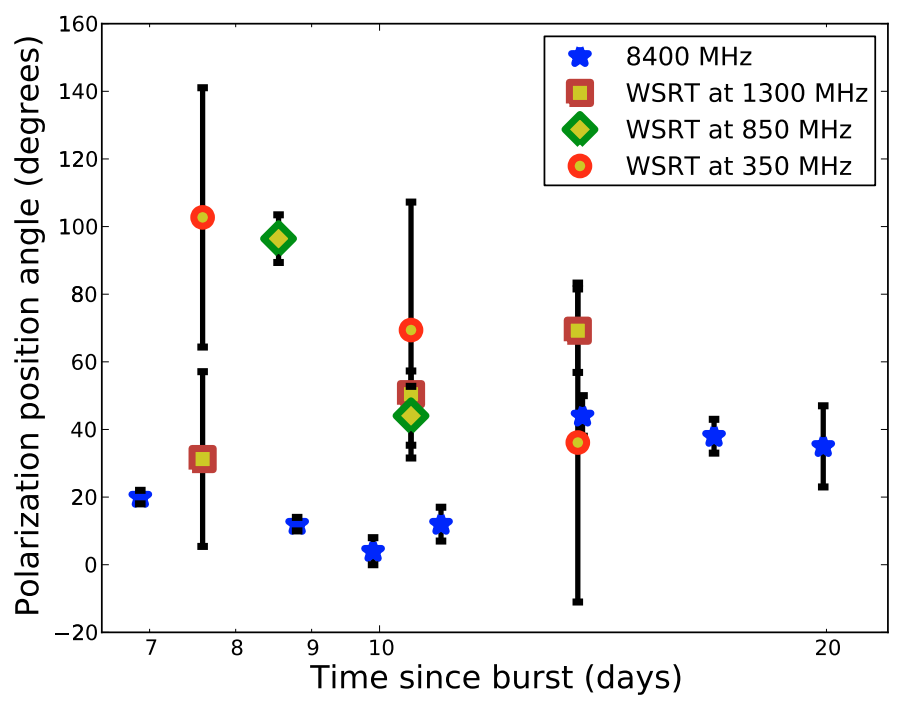

Fig. 4. Comparison between the polarization angles at 350, 850, 1300 and $8400 \mathrm{MHz}$

Table 2). In Fig. 4 we have done the same for the polarization angles. It is clear that the observations at $8.4 \mathrm{GHz}$ are much more accurate. Still, we do not see any significant discrepancies in the polarization fractions.

Our observations reveal larger polarization position angles than the $8.4 \mathrm{GHz}$ observations. Most compelling are the observations on 2005 january 5 at $850 \mathrm{MHz}$ and on january 7 at both 850 and $1300 \mathrm{MHz}$. The error bar on the polarization angle at $350 \mathrm{MHz}$ on january 4 is rather large, but this measurement and the $850 \mathrm{MHz}$ measurement on january 5 show the largest differences with the $8.4 \mathrm{GHz}$ observation, about $85^{\circ}$. At these times, the polarization angles from the $8.4 \mathrm{GHz}$ observations suggest that the magnetic field in the emitting plasma is aligned preferentially along the axis of the radio source, on average (Gaensler et al. 2005a). Thus, the january 4 and 5 polarization angles at 350 and $850 \mathrm{MHz}$ indicate that the magnetic field in the emitting plasma that causes linearly polarized radiation at these low frequencies is close to perpendicular to the axis of the radio source, within $\simeq 20^{\circ}$. Possibly a different substructure in the radio nebula is being probed. It seems hard to explain this feature without a complex model of the radio source.

\section{Conclusions}

It is striking that depolarization at low frequencies is absent. Also, we have shown that low frequency polarimetry of SGR
1806-20 provides hints with respect to the detailed substructure of the radio nebula which cannot be derived from the extrapolation of high frequency measurements. Models for the radio nebula need to take into account a distinct source of linearly polarized low frequency radiation with magnetic fields in the emitting plasmas aligned quite differently from the fields that are associated with radiation at high frequencies.

Acknowledgements. We thank Michiel Brentjens, James Miller-Jones and Gianni Bernardi for helpful discussions about polarization calibration. We thank Eric Greisen for providing background information about many AIPS tasks. The Westerbork Synthesis Radio Telescope is operated by ASTRON (Netherlands Foundation for Research in Astronomy) with support from the Netherlands Foundation for Scientific Research (NWO). This research was supported by NWO Vici grant 639.043.302 (HS and RAMJW) and by NWO NOVA project 10.3.2.02 (BS).

\section{References}

Abbott, D. C., Bieging, J. H., \& Churchwell, E. 1981, ApJ, 250, 645 Baars, J. W. M., Genzel, R., Pauliny-Toth, I. I. K., \& Witzel, A. 1977, A\&A, 61, 99

Blandford, R. D. 2005, in BAAS, 37, 1220

Brentjens, M. A. 2008, A\&A, 489, 69

Brown, S., \& Rudnick, L. 2009, AJ, 137, 3158

Cameron, P. B., Chandra, P., Ray, A., et al. 2005, Nature, 434, 1112

Chandra, P. 2005a, GRB Coordinates Network, 2947, 1

Chandra, P. 2005b, GRB Coordinates Network, 2995, 1

Condon, J. J., Cotton, W. D., Greisen, E. W., et al. 1998, AJ, 115, 1693

Dai, Z. G., Wu, X. F., Wang, X. Y., Huang, Y. F., \& Zhang, B. 2005, ApJ, 629, L81

Duncan, R. A., \& White, S. M. 2002, MNRAS, 330, 63

Fender, R. P., Muxlow, T. W. B., Garrett, M. A., et al. 2006, MNRAS, 367, L6 Gaensler, B. M., Kouveliotou, C., Gelfand, J. D., et al. 2005a, Nature, 434, 1104 Gaensler, B. M., Kouveliotou, C., Wijers, R., et al. 2005b, GRB Coordinates Network, 2931, 1

Gelfand, J. D., Lyubarsky, Y. E., Eichler, D., et al. 2005, ApJ, 634, L89

Greisen, E. W. 2003, in Astrophysics and Space Science Library, ed. A. Heck, 285,109

Hurley, K., Boggs, S. E., Smith, D. M., et al. 2005, Nature, 434, 1098

Israel, G. L., Belloni, T., Stella, L., et al. 2005, ApJ, 628, L53

Kettenis, M., van Langevelde, H. J., Reynolds, C., \& Cotton, B. 2006, in Astronomical Data Analysis Software and Systems XV, ed. C. Gabriel, C. Arviset, D. Ponz, \& S. Enrique, ASP Conf. Ser., 351, 497

Nakar, E., Piran, T., \& Sari, R. 2005, ApJ, 635, 516

Palmer, D. M., Barthelmy, S., Gehrels, N., et al. 2005, Nature, 434, 1107

Panagia, N., \& Felli, M. 1975, A\&A, 39, 1

Rea, N., Israel, G., Covino, S., et al. 2005, The Astronomer's Telegram, 645, 1 Schwartz, S. J., Zane, S., Wilson, R. J., et al. 2005, ApJ, 627, L129

Skinner, C. J., White, R. L., Becker, R. H., et al. 1996, in Radio Emission from the Stars and the Sun, ed. A. R. Taylor \& J. M. Paredes, ASP Conf. Ser., 93, 23

Tanvir, N. R., Chapman, R., Levan, A. J., \& Priddey, R. S. 2005, Nature, 438, 991

Taylor, G. B., Gelfand, J. D., Gaensler, B. M., et al. 2005, ApJ, 634, L93

Wang, X. Y., Wu, X. F., Fan, Y. Z., Dai, Z. G., \& Zhang, B. 2005, ApJ, 623, L29

Wright, A. E., \& Barlow, M. J. 1975, MNRAS, 170, 41 